\title{
Response to Robert G. Resta Commentary (Unprepared, Understaffed, and Unplanned: Thoughts on the Practical Implications of Discovering New Breast and Ovarian Cancer Causing Genes)
}

\author{
Wendy R. Uhlmann
}

Received: 4 August 2009 / Accepted: 12 August 2009 / Published online: 2 October 2009

(C) National Society of Genetic Counselors, Inc. 2009

Resta (2009) in his Commentary clearly articulates how another breast cancer gene discovery would significantly impact the genetic counselors' workload and appropriately urges us to think about these issues and be proactive. But a hypothetical BRCA3 gene is just the tip of the iceberg. The tsunami is genomic medicine, testing for common, complex conditions and personalized medicine. Are we ready to respond to patients' requests to order these tests, evaluate data or interpret reports from whole genome scans?

For the hypothetical BRCA3 gene, Resta (2009) proposes that we proactively develop protocols for recontacting patients that can be implemented with short notice, set up clinic databases with pedigrees and genetic test results to efficiently identify patients who need to be recontacted and then create mail merge letters. I think timewise and logistically, this is not practical. As Resta (2009) has already pointed out in his Commentary, we are doing all that we can to keep up with our current caseloads. Recontacting past patients would simply be relegated to the bottom of the perpetual "to do" list of tasks for patients, behind the insurance letters that have to be written to justify needed genetic testing. Given technology capabilities and electronic medical records, just like mammogram reminders are increasingly being used, this could similarly be implemented for a number of medical and genetic conditions. However, while re-contacting patients would be globally beneficial in healthcare, the fact is that we currently do not put this responsibility on other physicians and there are both significant time and logistical issues.

\section{W. R. Uhlmann ( $\bowtie)$}

Division of Molecular Medicine and Genetics,

University of Michigan Health System,

300 North Ingalls, NI3 A03,

Ann Arbor, MI 48109-5419, USA

e-mail: wuhlmann@umich.edu
But there are alternatives. First, put the onus on the patient to re-contact the clinic to inquire whether there have been any updates in genetic testing and treatment since their appointment and include this instruction in visit documentation. Second, encourage patients to join, visit the websites or call genetic support groups since these groups will often have up-to-date information about advances in care and genetic testing. Third, time to re-contact patients could instead be used to create and update clinic websites with pertinent information.

Patients could access the clinic website pre and post their appointments for information including basic genetic concepts, patterns of inheritance, genetic conditions (e.g. Genetics Home Reference), genetic testing and factors to consider, insurance issues and links to support group directories. I propose that GeneTests develop a page that clinics could link to that would list genetic conditions, indicate if genetic testing is available and last date there was a significant change in testing. The clinic website would include a standard disclaimer about use of web information and the need to make medical decisions in consultation with a physician along with a statement emphasizing that a genetic test does not take the place of a genetic evaluation and that there are several factors to consider. Patients previously seen could easily use the clinic website to look up the genetic condition, determine if genetic testing has changed since their visit and initiate contact with the clinic. For patients with no computer, a call to the clinic or use of public library computers would be encouraged. The fact is, patients are using the Internet to obtain genetic testing information and some are even having direct-to-consumer genetic testing.

Resta (2009) states that "...whatever testing is done will likely entail a DNA sample and plenty of explanation." Many tests are done in our healthcare without "plenty of 
explanation." As Resta (2009) points out, "Many patients will only vaguely remember the initial counseling details"; I agree. In order to enhance understanding and avoid information overload, we need to rethink the information that needs to be conveyed so that risks are understood and informed decisions can be made about genetic testing and healthcare. Is a genetics mini-lesson on chromosomes, genes etc. a necessary part of a clinic visit? Could we provide patients with an information sheet before or during the clinic visit or is such an explanation even needed? Patients often want to know their bottom-line risk and are less interested in how the risk figure was derived. When newborn screening is done, there is not a detailed explanation of the incidence and pattern of inheritance of each condition. It is sufficient to know that these conditions have increased morbidity and mortality and for some conditions, early treatment is of benefit. We need to work on determining when genetic testing merits more discussion (e.g. predictive genetic testing; genetic tests with low sensitivity; tests with complex interpretations etc.) versus when it can be done like newborn screening or a CBC. There will be an increasing number of genetic tests for common complex conditions and in tests that will just be risk modifying. I agree with Resta (2009) that genetic tests should be rigorously evaluated before introduction into clinical practice and to discuss DNA banking with patients. We should also encourage specialists and primary care physicians to discuss this option in cases where lifespan is limited.

Genetic clinic visits have typically focused on a single indication. With the era of genomic medicine, the focus will shift from uncommon Mendelian inherited conditions to addressing a number of genetic conditions during a clinic visit. We need to rethink the time involved and what can be accomplished in clinic visits (traditional visits are often an hour or more), and recognize that more than one clinic visit will be necessary to address all genetic issues, just like standard medical care. To keep the counseling in genetic counseling, we need to have time to raise and address psychosocial issues and not defer this as a discussion only if time permits. Resta (2009) states that "Complicated psychological and emotional issues will often emerge." While such issues can be complicated, there are a number of patients who just want the information or "the test" with no psychosocial exploration. However, it is important that we give patients the opportunity to have psychosocial issues discussed, and provide helpful resources.

We need to globally think about developing other genetic service delivery models, including having patients enter family history information by computer at home or in the waiting room with electronic pedigree generation, group education sessions (and use of CD-ROMS, videos, computer programs) followed by individual genetic counseling for commonly seen conditions and other changes. With all models (and points in this Commentary), we need to consider options for underserved populations, patients with learning disabilities, physical disabilities and language barriers.

With the era of genomic and personalized medicine, everyone in the population is potentially a patient. Therefore, there will be a "flood" of patients who will need genetic issues addressed and genetic testing but not all of these patients need to be seen in genetics clinics. Resta (2009) states that if patients cannot be seen in a timely manner in genetics clinics, "this increases the likelihood that patients will be inadequately counseled by non-genetic specialists and that test results will be inaccurately interpreted." This statement is simply not true and incorrectly implies that only geneticists can accurately interpret genetic test results. Genetic counselors and geneticists do need to be proactive in developing triaging guidelines and genetic testing algorithms and seek input from specialists and primary care physicians. Which are the patients who should be seen in the genetics clinic versus by a specialist or by a primary care physician? What resources and guidelines can we provide primary care physicians so that they will know when referral is indicated and when genetic testing should be ordered versus deferred to a specialist or genetics clinic? This has been successfully done with maternal serum screening during pregnancy where testing is ordered by obstetricians and patients with abnormal values are often referred to prenatal genetics clinics. We are experts in genetics and clinical care applications and need to continue to share our expertise, including publishing articles in the medical literature, developing resources for physicians and patients, giving presentations and serving on committees.

As we transition from Mendelian conditions to genomic medicine, common complex diseases and personalized medicine, we need to:

1) Keep educating each other through online courses, presentations at our Annual Education Conferences and regional meetings, journal publications and resources at our website.

2) Think globally about models of providing genetic services and recognize that additional models need to be developed. We need to focus on key components of genetic counseling including risk assessment and communication, education about the genetic condition, facilitation of decision-making, addressing psychosocial issues and providing supportive resources.

3) Change our standards for how we document traditional genetics clinic visits. Letters can sometimes take as long to compose as the clinic visit itself. Rather than generate $3+$ page summaries of clinic visits that explain 
chromosomes, genes, patterns of inheritance, risk figures and their derivations, instead give bottom-line summary statements and cite information sheets and the clinic website for further explanation of basic genetic concepts. Many clinics do use template letters however these letters are often still long.

4) Collaboratively develop triaging guidelines and testing algorithms. Develop guidelines and points to consider for types of genetic tests (e.g. diagnostic, carrier, predictive) since development of this information for each genetic test will take years. NSGC's Special Interest Groups (SIGs) can initiate developing guidelines for genetic conditions in their specialty area.

5) Continue to educate insurers about the value of genetic testing, implications for family members and potential savings to our healthcare system. For genetic conditions that require screening with echocardiograms, MRIs or CT scans, the cost of any of these tests, done even once, will far exceed the cost of most genetic tests. Healthcare dollars can be saved if patients, who are not at increased risk by genetic testing, no longer undergo costly screening.
6) Teach patients to be educated seekers of information. Put the onus on patients to contact genetic support groups/visit websites and re-contact the genetics clinic to learn about advances in treatment and genetic testing for their genetic condition.

I agree with Resta (2009) that "professional, patient, industry and government organizations must work together." In order to provide the best care, we need to reassess the services to provide, what information needs to be communicated, what is best for us to do and what can be done by other healthcare professionals and even by patients themselves. The genomic train has already left the station and we need to do what we can to keep it on the right track.

\section{Reference}

Resta, R. G. (2009). Commentary. Unprepared, uderstaffed and unplanned: Thoughts on the practical implications of discovering new breast and ovarian cancer causing genes. Journal of Genetic Counseling, 18(6). 